\title{
Laparoscopic repair of inguinal hernia in adults
}

\author{
Xue-Fei Yang ${ }^{1}$, Jia-Lin Liu ${ }^{2}$ \\ ${ }^{1}$ Department of Surgery, The University of Hong Kong-Shenzhen Hospital, the University of Hong Kong, Shenzhen 518053, China; ${ }^{2}$ Department \\ of Hepatobiliary Surgery, Shenzhen People's Hospital \& Second Clinical Medical College of Jinan University, Shenzhen 518020, China \\ Correspondence to: Jia-Lin Liu. Department of Hepatobiliary Surgery, Shenzhen People's Hospital, No. 1017, North Dongmen Road, Luohu District, \\ Shenzhen 518020, China. Email: szliujialin@126.com or 4683755@qq.com.
}

\begin{abstract}
Laparoscopic repair of inguinal hernia is mini-invasive and has confirmed effects. The procedures include intraperitoneal onlay mesh (IPOM) repair, transabdominal preperitoneal (TAPP) repair and total extraperitoneal (TEP) repair. These procedures have totally different anatomic point of view, process and technical key points from open operations. The technical details of these operations are discussed in this article, also the strategies of treatment for some special conditions.
\end{abstract}

Keywords: Inguinal hernia; laparoscopic repair; intraperitoneal onlay mesh (IPOM); transabdominal preperitoneal (TAPP); total extraperitoneal (TEP); mesh; fixation

Submitted Aug 29, 2016. Accepted for publication Sep 21, 2016.

doi: $10.21037 / \mathrm{atm} .2016 .10 .37$

View this article at: http://dx.doi.org/10.21037/atm.2016.10.37

Laparoscopic repair of inguinal hernia is mini-invasive and has confirmed effects (1-4). The procedures include intraperitoneal onlay mesh (IPOM) repair, transabdominal preperitoneal (TAPP) repair and total extraperitoneal (TEP) repair. These procedures have totally different anatomic point of view, process and technical key points from open operations. Surgeons must understand the basic principles, anatomic key points and the risks of these operations to insure the surgical effects and prevent complications before performance. The experiences to deal with some special conditions are also very important to the safety and results.

\section{Indications}

Laparoscopic inguinal hernia repair is well suited for all types of adult inguinal hernia, especially bilateral hernias and recurrence after open hernia repair. However, the laparoscopic approach for inguinal hernia repair should be used with caution in patients with a history of lower abdominal surgery, abdominal radiotherapy, or bleeding tendency or in patients with giant irreducible hernia.

\section{Contraindications}

The contraindications are as follows: inability to tolerate general anesthesia, severe benign prostatic hyperplasia, cirrhotic ascites, acute incarcerated or strangulated hernia, and skin infection on the lower abdominal wall.

\section{Surgical options}

Common laparoscopic approaches for inguinal hernia repair in adults include three options: IPOM repair, TAPP repair and TEP repair. The basic principle for the hernia repair, i.e., repairing the weakness of the myopectineal orifice, is the same in all three approaches. The IPOM and TAPP techniques repair the hernia intraperitoneally, while the TEP approach requires the establishment of pneumopreperitoneum. It is easier to find bilateral, complex and occult hernias using the TAPP approach because of the access to the abdominal cavity. This approach is especially suited for incarcerated hernia and irreducible hernia. Given various surgical difficulties, the requirements for peritoneum integrity and properties of the selected mesh in the IPOM, TAPP and TEP repair techniques, the surgical indications for the three approaches are accordingly different. Beginners can choose to perform the TAPP repair to reduce the surgical difficulty and to quickly master groin applied anatomy and surgical skills. With more experience, the surgeon can choose to perform TEP repair, which has 
many advantages, including not only the lower incidence of abdominal adhesions but also reduced expense. In patients with large scrotal hernia, a history of lower abdominal surgery or recurrent hernia, the hernia sac can be difficult to dissect; additionally, the extraperitoneal space might have been compromised by the previous surgeries. In such cases, the TAPP approach is the optimal selection. The IPOM approach may be selected for cases in which the above-mentioned two approaches cannot be used. In patients with a specific need to protect the spermatic cord, a history of lower abdominal radiotherapy, multiple recurrent hernia and heart and lung conditions that do not allow prolonged surgery, the IPOM approach can be selected, not only to avoid the risk of spermatic cord damage and difficult dissection of the peritoneum but also to shorten the operative time. However, the IPOM approach is not the most preferred method for laparoscopic surgery. To prevent postoperative abdominal adhesions, anti-adhesive mesh must be used in the IPOM approach.

\section{Preoperative evaluation}

Medical histories are collected to determine whether conditions that can induce increases in intra-abdominal pressure, such as chronic coughing, chronic constipation or difficulty urinating, are present, as increased intraabdominal pressure is a common cause of postoperative hernia recurrence. In senior male patients, the history of nocturia should be obtained to evaluate if benign prostatic hypertrophy is present; if necessary, a post-void residual urine test may be performed. In male patients aged 50 and older, a selective $\alpha-1$ receptor blocker, such as tamsulosin, should be prescribed orally ( 2 or 3 times daily) 2 or 3 days before surgery. This regimen can significantly reduce the incidence of postoperative acute urinary retention. Respiratory infection should be controlled before surgery, which can be scheduled after the coughing is relieved. For patients with recurrent hernia, the history of previous procedures (particularly of the use of synthetic mesh) and their postoperative diagnoses should be obtained to accurately assess the difficulty of the surgery and to select the appropriate surgical procedures. Preoperative examination in male patients should include careful examination of the testicles, such as size, texture and location. Ultrasonography should be routinely performed in the bilateral inguinal region to exclude cryptorchidism, spermatic cord hydrocele and tumors.

\section{Anesthesia}

General anesthesia is commonly administered with an endotracheal tube. A laryngeal mask may be used with the anesthesia to reduce severe coughing caused by stimulation from tracheal intubation and to protect the effectiveness of the hernia repair upon awakening.

\section{Surgical preparation}

The skin of the abdomen and the umbilicus should be prepared according to laparoscopic surgery requirements. Preoperative prophylactic antibiotics are given 30 minutes before surgery to reduce the risk of infections in high-risk groups (advanced age, diabetes, obesity, weight loss, multiple recurrent hernias, recent chemoradiotherapy or other immunocompromised conditions). Patients are instructed to empty their bladder before surgery. Placement of the urethral catheter is not required if the expected operative time is not long in experienced hands. However, if a large residual urine volume is noted during surgery, a urethral catheter can be placed to obtain better exposure of the operative field and can be removed at the end of the surgery.

\section{Surgical positioning}

Patients are placed in a supine position. Considering the fact that the bowel loops located in the pelvis or iliac fossa may affect exposure of the inguinal region, the operating table may need to be adjusted during surgery to place patients in the Trendelenburg position $\left(10^{\circ}-15^{\circ}\right)$ to facilitate exposure of the inguinal region and to protect the bowel loops from injury.

\section{Required equipment and instruments}

Standard laparoscopic equipment is required as follows: a $30^{\circ}$ laparoscope, a monopolar or bipolar coagulation device (one each), a 5 -mm ultrasound dissector, a $10-\mathrm{mm}$ trocar, two 5-mm trocars, a 5-mm scissors, a 5-mm non-traumatic clamp, a 5-mm grasper, a 5-mm needle holder, a 5-mm laparoscopic spiral tack applier (multiple tacks), 15×[10-15]$\mathrm{cm}^{2}$ polypropylene mesh, and $[12-15] \times[8-10]-\mathrm{cm}^{2}$ antiadhesive mesh.

\section{Operating room layout}

A video monitor is placed at the patient's feet. The surgical 

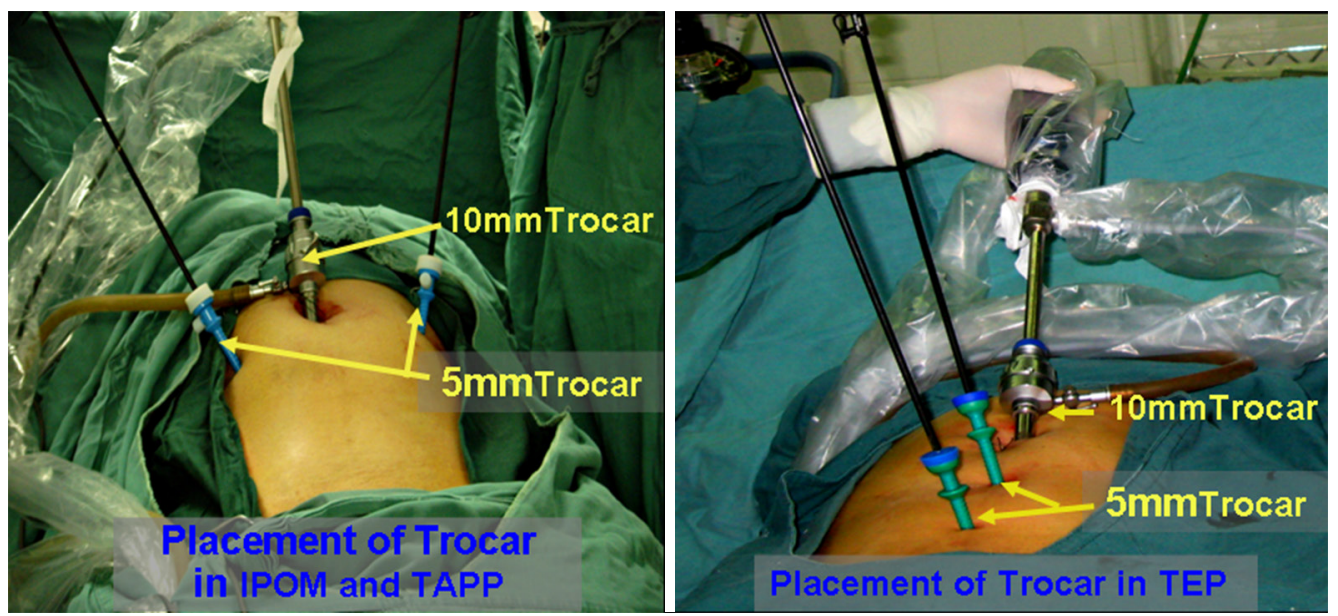

Figure 1 Trocar placement. IPOM, intraperitoneal onlay mesh; TAPP, transabdominal preperitoneal; TEP, total extraperitoneal.

assistant stands on the patient's affected side, and the surgeon stands on the side opposite to the assistant. In terms of surgeries for patients with bilateral inguinal hernia, the surgeon may stand on either side based on their preference. The surgeon may change location with the surgical assistant at any time when appropriate. The anesthetist stands at the patient's head. The instrument nurses stand in line with the surgeon.

\section{Trocar placement}

Trocar placement may vary according to the surgical procedure, the operating needs and the surgeon's preference. For performing IPOM and TAPP repairs, the trocars may be placed in same pattern. A $10-\mathrm{mm}$ trocar is placed below the umbilicus; two 5-mm trocars are placed at the lateral edge of the rectus abdominis at the umbilical level on each side of the abdomen. For TEP repair, one periumbilical $10-\mathrm{mm}$ trocar and two $5-\mathrm{mm}$ midline trocars (in a point of trisection of the connecting line between the umbilicus and the pubic symphysis) are used (Figure 1). The three trocars are in line with each other. The surgeon may find this arrangement to be odd in the early stage of the "learning curve", but it is actually helpful for performing bilateral inguinal hernia repair. If necessary, one $5-\mathrm{mm}$ trocar close to the pubic symphysis may be relocated to a point medial-superior to the anterior superior iliac spine on the affected side to facilitate the surgeon's manual operation with both hands. As the surgeon's skill improves, two-port or even single-port laparoscopic approaches may be chosen for the hernia repair. However, a platform for a single-port laparoscopic approach or a laparoscope with a working port and specific surgical instruments are required.

\section{Conventional laparoscopic exploration}

During the TAPP and IPOM procedures, conventional laparoscopic exploration should be performed after the laparoscope is inserted into the abdominal cavity. This exploration should cover several areas. (I) It should be noted if large amounts of abdominal vessel and/or bowel damage are present during the establishment of pneumoperitoneum and trocar placement and if all major abdominal organs are normal; (II) the bilateral inguinal region and important structures should be observed to confirm the diagnosis of indirect inguinal hernia, direct inguinal hernia or femoral hernia; it should be noted that femoral hernia is easily missed because of the smaller diameter of the hernia ring; (III) it should also be noted if the hernia sac contains the omentum, bowel loops, fallopian tubes or other organs. If so, they need to be reduced completely; (IV) the presence of occult inguinal hernia should be confirmed by pressing the groin area with a hand. Unformed direct hernia is a major type of occult inguinal hernia in adults. When the surgeon presses the groin area toward the inside of the abdominal cavity, the extraperitoneal fat tab can be seen to protrude into the abdominal cavity from the defect of the weak transverse. The protrusion indicates the position of the occult direct inguinal hernia; (V) larger spermatic cord lipoma may also cause occult indirect inguinal hernia and manifests similarly as occult direct inguinal hernia (Figure 2). 

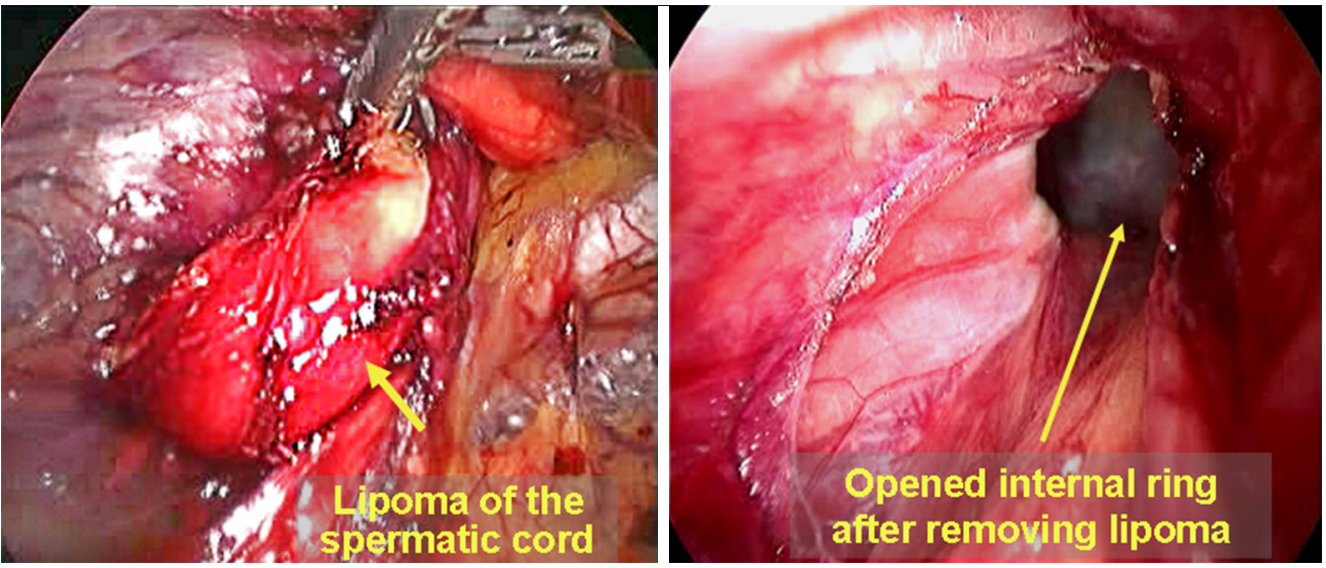

Figure 2 Spermatic cord lipoma.

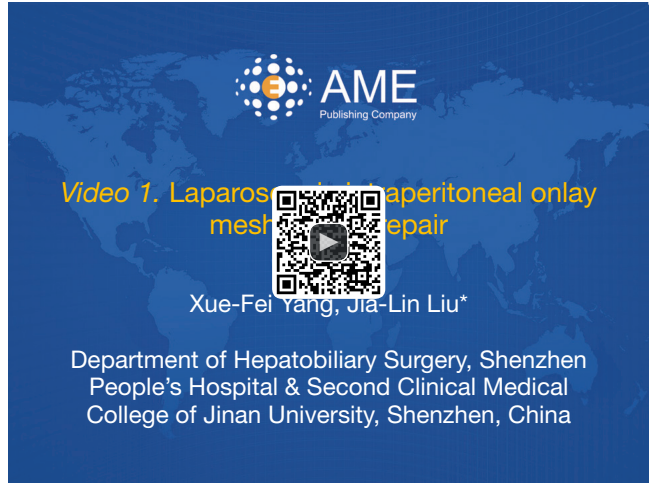

Figure 3 Laparoscopic intraperitoneal onlay mesh (IPOM) repair (5). Available online: http://www.asvide.com/articles/1174

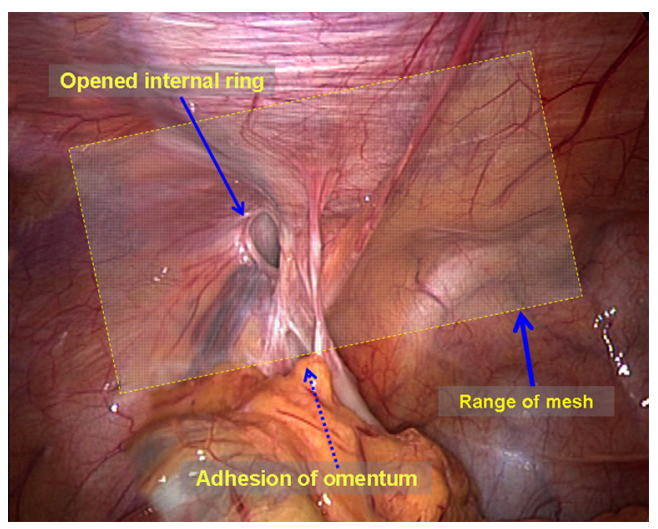

Figure 4 The area for mesh placement.

\section{Surgical procedures}

\section{Laparoscopic IPOM approach (Figure 3)}

The surgical area is prepared and draped as normal. A $10-\mathrm{mm}$ skin incision is made along the lower edge of the umbilicus. A small retractor is used to expose the midline. A Veress needle is inserted into the abdominal cavity to create pneumoperitoneum, and the pressure is maintained at 12-14 mmHg. Then, a $10-\mathrm{mm}$ trocar and a $30^{\circ}$ laparoscope are placed for exploration. A $5-\mathrm{mm}$ skin incision is made in the lateral edge of the rectus abdominis at the umbilical level on each side of the abdomen for a 5-mm trocar and surgical instruments. The above description is for a closed establishment of pneumoperitoneum. The open establishment of pneumoperitoneum can also be used based on the surgeon's preference and the patient's condition (for example, history of abdominal surgery).

Dissection of the mesh placement area. The omentum or the organs in the hernia sac are grasped using a nontraumatic clamp and are moved back into the abdominal cavity. The peritoneum around the myopectineal orifice is exposed clearly. All adhesion structures, including the ileocecal portion and the sigmoid colon, are separated to the cephalad side (Figure 4). At level of the Cooper ligament, the medial umbilical fold is incised horizontally to facilitate flat placement of the mesh. Diathermy scissors are then used to incise the closed umbilical artery; if bleeding is significant, it should be ligated (Figure 5). When the area around the myopectineal orifice is dissected completely, the mesh can be 


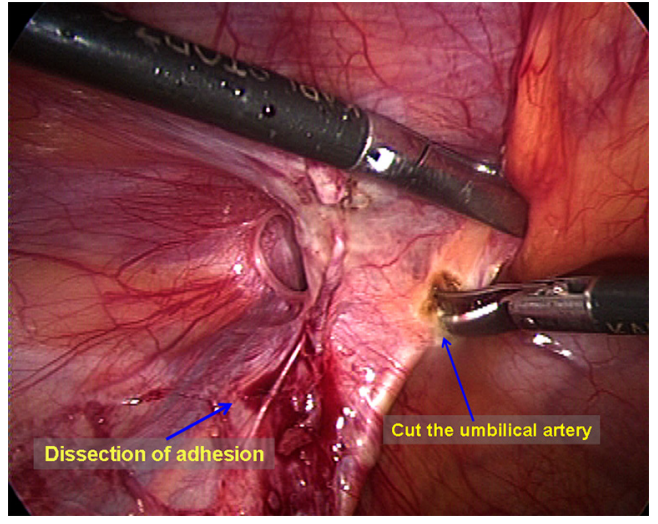

Figure 5 The medial umbilical fold is incised, and the umbilical artery is divided.

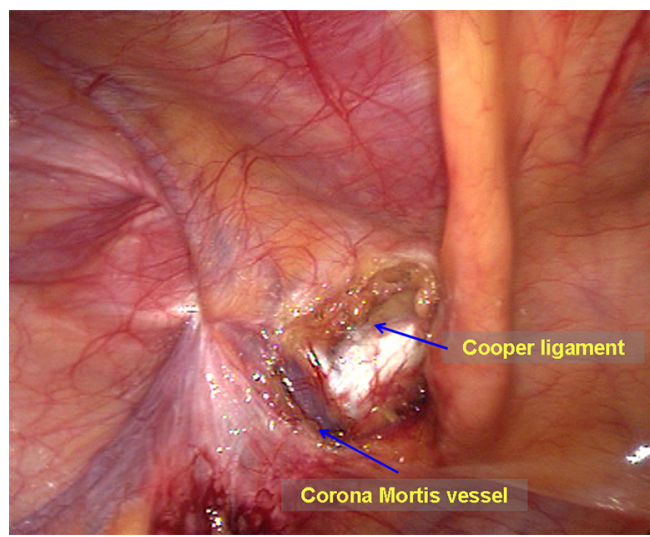

Figure 6 Exposure of the Cooper ligament and the Corona Mortis vessels.

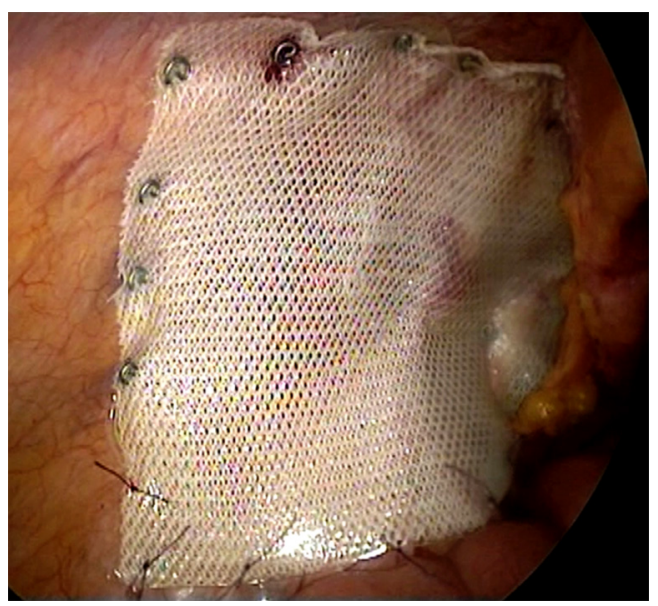

Figure 7 Diagram of a completed mesh fixation. placed at this time. In some patients, the peritoneum and the fat layer of the pubis surface are too thick. In such cases, it is difficult to determine the location of the Cooper ligament and the Corona Mortis vessels. To avoid damage to blood vessels when placing spiral tacks, the parietal peritoneum can be incised at the site whereby the umbilical fold was incised to expose the Cooper ligament and the Corona Mortis vessels (Figure 6) and to separate medially until the pubic symphysis is reached. It is recommended to incise the medial umbilical fold when performing an IPOM repair so that the mesh is exactly fixed to the Cooper ligament with care to avoid mesh migration or compression on the bladder wall.

Mesh placement and fixation. The laparoscope is withdrawn from the abdominal cavity. A sheet of antiadhesion mesh $\left\{[12-15] \times[8-10] \mathrm{cm}^{2}\right.$ in size $\}$, which is rolled into a cigarette-like roll, is introduced through the $10-\mathrm{mm}$ port into the abdominal cavity, and the laparoscope is then placed into the abdominal cavity again. The long axis of the mesh should lie parallel with the iliopubic tract. The mesh is then unfolded, and the medial edge is pushed out until the pubic symphysis is reached, covering the Hesselbach triangle, the femoral hernia ring and the indirect hernia ring. The unfolded edge of the mesh should cover the defect with $3 \mathrm{~cm}$ extending beyond its edge. The antiadhesion surface of the mesh should face the abdominal cavity. The mesh is initially fixed to the pubic tubercle and the Cooper ligaments with a spiral tack. The spiral tack on the Cooper ligament should not go beyond $5 \mathrm{~cm}$ lateral to the pubic symphysis to avoid damage to the Corona Mortis vessels. The lateral portion of the mesh is then unfolded flat; the tacks are tacked along the upper edge of the mesh at intervals of 3 to $4 \mathrm{~cm}$ until the lateral edge of the mesh is reached. Care is taken to ensure that the tacks cannot be placed at spots lower than the iliopubic tract and to ensure not to injure the vessels when placing tacks along both sides of the inferior epigastric vessels. In IPOM repair, the mesh is usually fixed to the pubic symphysis, the Cooper ligaments and the lower abdominal wall. The portion of the mesh arching across the iliac vessels does not need to be fixed to the peritoneum. However, to prevent postoperative mesh migration or herniation of the bowel loops (resulting in recurrence of hernia), it is recommended that mesh fixation be performed under the laparoscope with interrupted suture. Care must be taken not to damage the blood vessels and the nerves during suturing (Figure 7). The medial umbilical incision does not need to be sutured to the 


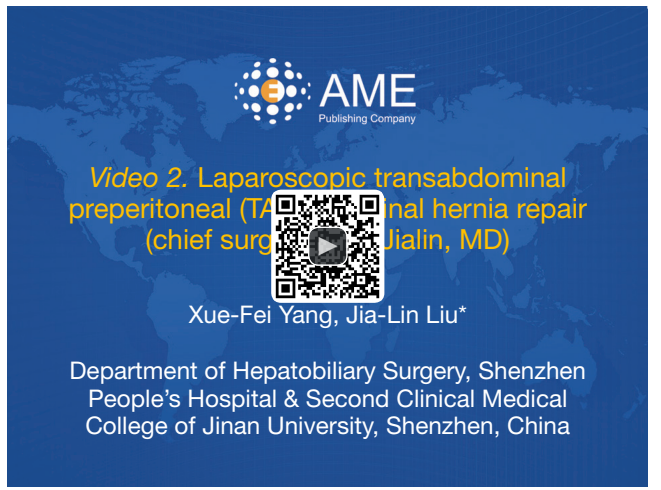

Figure 8 Laparoscopic transabdominal preperitoneal (TAPP) inguinal hernia repair (chief surgeon: Liu Jialin, MD) (6). Available online: http://www.asvide.com/articles/1175

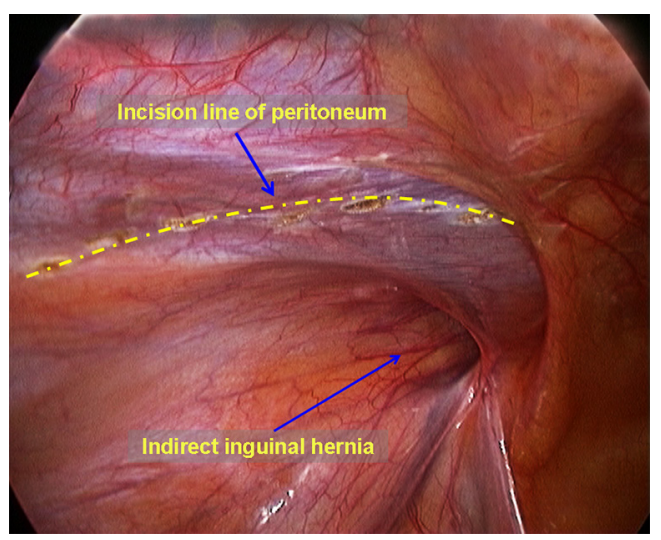

Figure 9 The peritoneum is incised with a curved incision.

anti-adhesion mesh.

\section{Laparoscopic TAPP inguinal hernia repair (Figure 8)}

The surgical area is prepared and draped as normal. Pneumoperitoneum is established according to the method described in the section summarizing the IPOM approach. The pressure is maintained at $12-14 \mathrm{mmHg}$. Then, a $10-\mathrm{mm}$ trocar and a $30^{\circ}$ laparoscope are placed to explore the bilateral inguinal region to confirm the diagnosis and to reduce the hernia contents.

Incising the peritoneum and initially dissecting the preperitoneal space. A left indirect inguinal hernia is taken as an example in this section. Diathermy scissors/ ultrasonic dissector were used to incise the peritoneum with a curved incision from the root of the medial umbilical folds to the anterior superior iliac spine (Figure 9). The edge of the peritoneum is then grasped using a grasper and diathermy scissors, or another grasper is used to separate the retropubic preperitoneal space, the space of Bogros, and the iliac fossa posterior-inferiorly in the extraperitoneal space between the peritoneum and the abdominal transverse fascia. It is relatively easy to separate the above-mentioned extraperitoneal space to medially reach the pubic symphysis to expose the pubic ramus and the Cooper ligament, with care taken to protect the bladder medial-inferior to the Cooper ligament, and to laterally reach the anterior superior iliac spine. Care should be taken to separate the peritoneum that covers the inferior epigastric vessels. In the space of Bogros, a sheet-like and thickening portion of the transverse fascia makes the transversus abdominis and the peritoneum tightly adhesive. During the dissection, these portions should be incised to gain initial access to the space of Bogros. Complete dissection of the space of Bogros and the iliac fossa are not performed until the hernia sac is dissected.

Dissection of the hernia sac. For indirect inguinal hernia sac dissection, it is easy to identify the hernia sac if searching along the Cooper ligament during dissection of the space of Retzius. The boundary line between the transverse fascia and the hernia sac comes into view after slightly dissecting the hernia sac. The hernia sac is grasped using a grasper and is separated free from the loose and deformed transverse fascia (pseudo sac). The hernia sac can be reduced at this stage. The dissection is now continued in the cephalad direction to separate the direct hernia sac from the Cooper ligament until the Cooper ligament and the direct hernia defect (Figure 10) are clearly exposed. A hematoma is easily formed after surgery if the direct hernia defect is relatively large. The loose pseudo-sac should be loop-ligated or pulled out to tack to the Cooper ligament. It is believed that this action reduces the incidence of a postoperative hematoma.

An indirect hernia sac is an extension of the peritoneum and is wrapped by the spermatic fascia with the vas deferens and the testicular vessels on its interior. This type of sac herniates into the inguinal canal at a site lateral to the root of the inferior epigastric vessels. Thus, one must incise the internal spermatic fascia at the vessel root to expose the white indirect hernia sac. Then, a blunt or sharp dissection is performed to separate the testicular vessels and the vas deferens from the hernia sac (Figure 11). If the indirect inguinal hernia sac did not completely herniate into the scrotum or only herniated very mildly, it can be easily and completely separated and reduced. However, in large hernia sacs (the sac herniates the scrotum deeply) or very adherent 

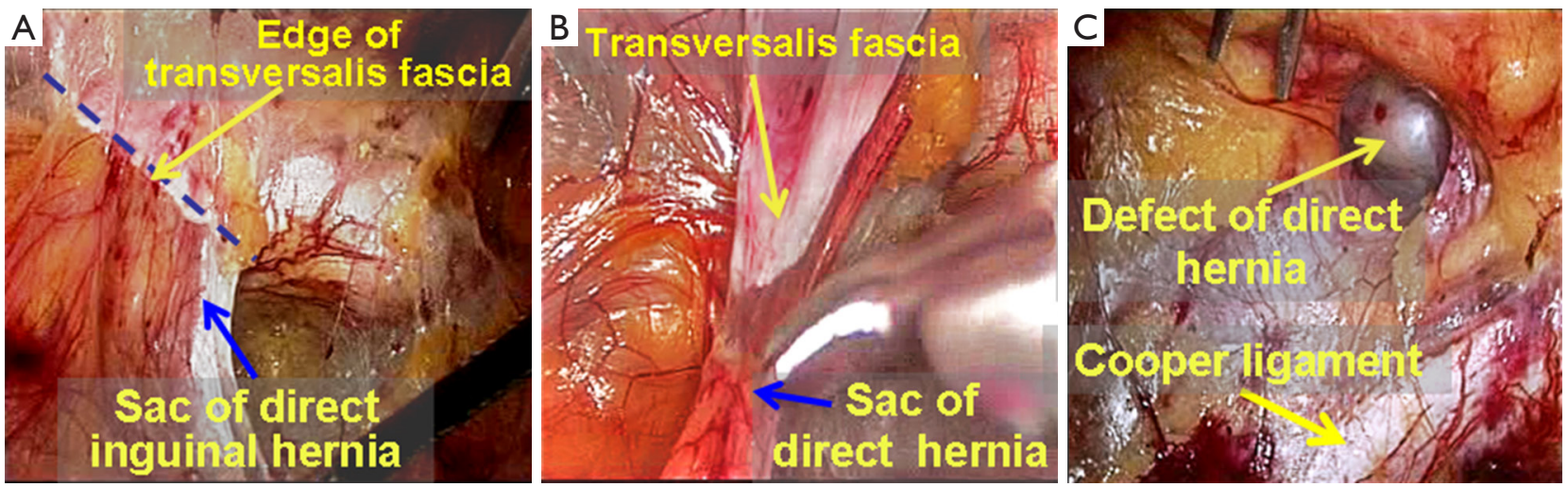

Figure 10 Dissection of the direct hernia sac.

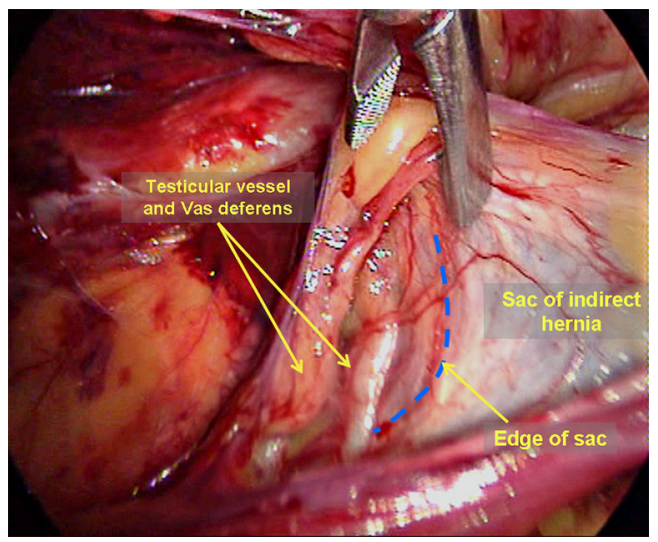

Figure 11 Dissection of an indirect inguinal hernia sac.

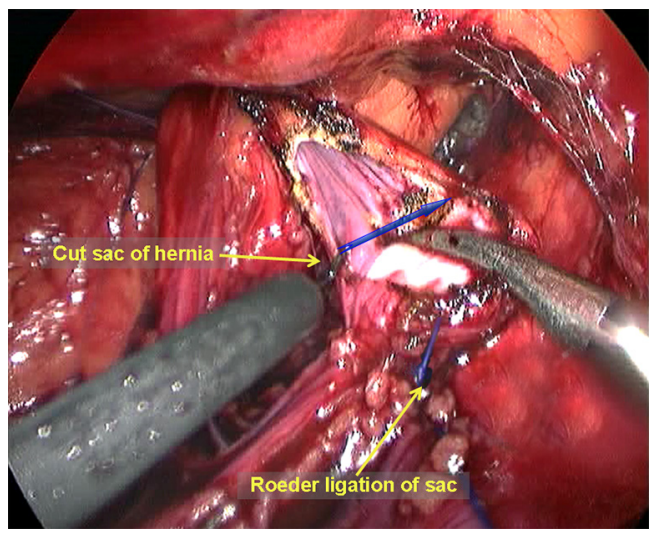

Figure 12 A diathermy hook is used to transect the hernia sac.

sacs (due to a longer disease course), it is not necessary to dissect or reduce the sacs completely; the sacs can be ligated by external or intra-abdominal ligation and then incised at the level of the internal ring (Figure 12). It is important to ensure proper hemostasis at the distal end of the sac.

Dissection of the proximal end of the hernia sac and the peritoneal folds, parietalization of the cord structures, and complete dissection of the space of Bogros. It is easy to view the peritoneal folds after transection of the hernia sac; the proximal end of the sac and the peritoneal fold are grasped and retracted to the cephalad side with a certain tension, and combined blunt and sharp dissection is then used to separate the peritoneal fold free from the testicular vessels and the vas deferens; this process is called clarification of cord structures (Figure 13). It is important to ensure that the distance from the peritoneal fold to the internal ring is $5 \mathrm{~cm}$ or more to facilitate the placement of the mesh and closure of the peritoneal incision. In male patients, it is very common to find that the vas deferens is encircled by the deep transverse fascia at the deep level, namely the preperitoneal loop. Transection of the preperitoneal loop can maximally benefit adequate parietalization of the cord structures and facilitate flat placement of the mesh against the spermatic cord (Figure 14). In female patients, the round ligament of the uterus is covered by the indirect hernia sac and dense adhesions, and dissection between them can be difficult. Therefore, the sac and the round ligament can be ligated together at a higher level; a mesh with a notch can be inserted later, and the distal portion of the sac is left open (Figures 15,16). The alternative is to incise a portion of the peritoneum adherent to the round ligament of the uterus to make a parietalization of the ligamentum teres uteri and then close the peritoneal incision with sutures to preserve the intact round ligament of the uterus. The dissection then continues laterally to dissect the peritoneal folds in the space of Bogros and the 
Page 8 of 19

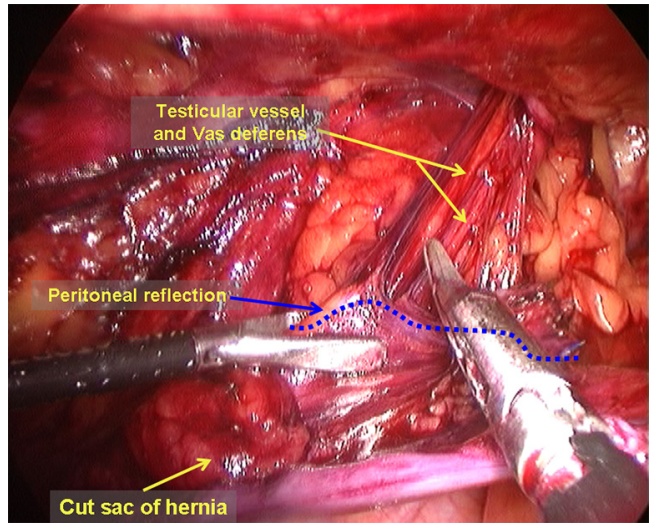

Figure 13 Clarification of cord structures.

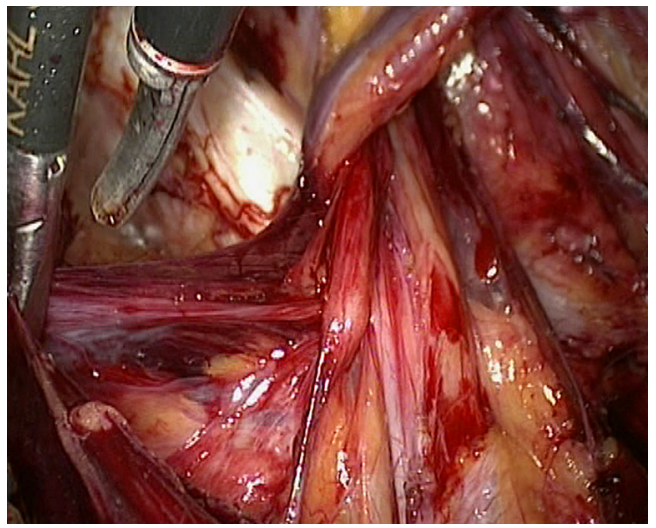

Figure 14 Preperitoneal loop.

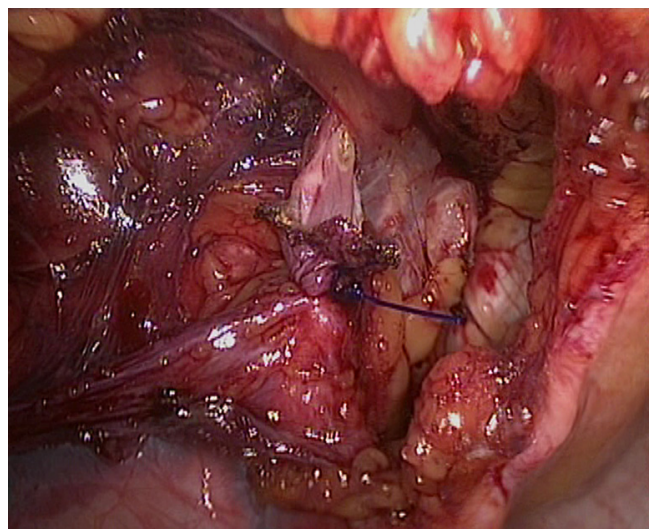

Figure 15 The distal portion of the sac is left open.

posterior abdominal fat tab toward the cephalad side to expose the iliac fascia of the posterior abdominal wall. Care should be exercised to avoid damaging the surface fascia

\section{Yang and Liu. Laparoscopic repair of adult inguinal hernia}

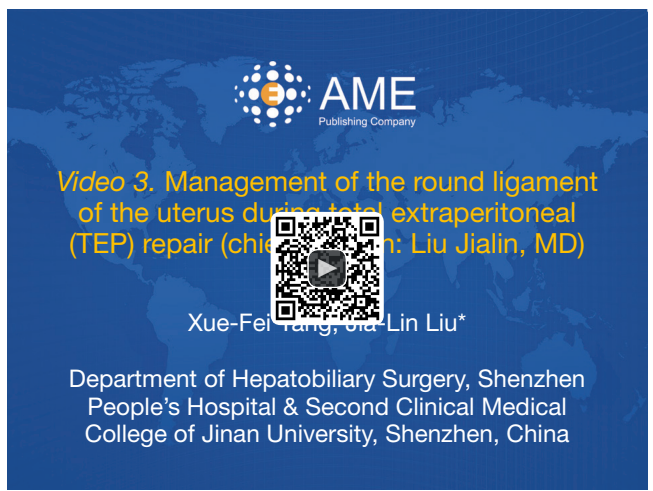

Figure 16 Management of the round ligament of the uterus during total extraperitoneal (TEP) repair (chief surgeon: Liu Jialin, MD) (7). Available online: http://www.asvide.com/articles/1176

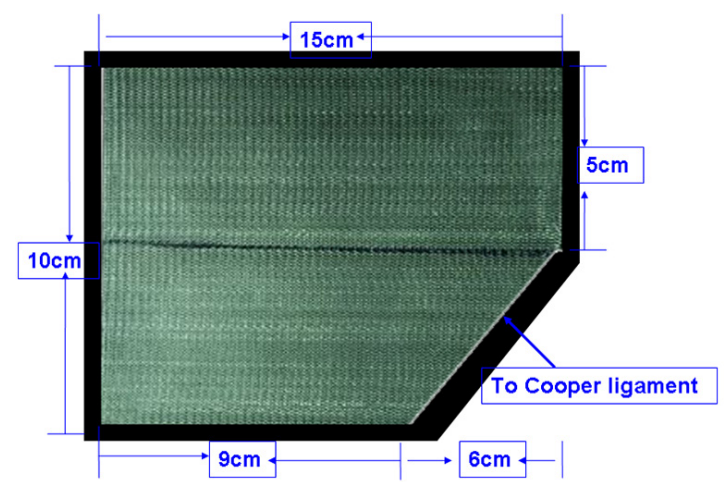

Figure 17 Tailoring of the polypropylene hernia mesh.

of the psoas muscle, the genitofemoral nerve, which runs inferiorly on the surface of this muscle, and the lateral femoral cutaneous nerve, which runs inferior-laterally along the edge of the psoas muscle. The goal is to generate enough space for the placement of the lateral portion of the mesh. Once these steps are completed, the dissection of the extraperitoneal space is completed;

Tailoring, placement and fixation of the mesh. A polypropylene hernia mesh is used as an example. A $[15-14] \times[12-10]-\mathrm{cm}^{2}$ mesh is created from a $15 \times 15-\mathrm{cm}^{2}$ piece of polypropylene mesh, shaped as shown in Figure 17. The medial corner of the mesh is cut to accommodate the anatomy of the pubic ramus and has a 1-cm overlap with it. Cutting a corner from the mesh can assist in recognizing its orientation and can help avoid bladder irritation from using over-sized mesh, which can cause paresthesia of voiding or erosion of the bladder. The mesh is then tightly rolled up (a beginner can encircle and tie the middle of the roll 


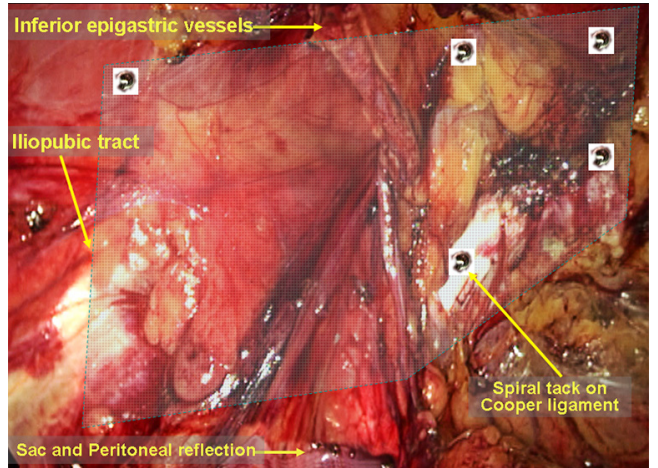

Figure 18 Schematic of mesh fixation.

with a suture to make it easier to handle) and passed, using a grasper, through the umbilical trocar into the abdominal cavity. The mesh roll is then placed in the extraperitoneal space and unrolled to the myopectineal orifice. The mesh should cover the conjoint tendon for $2-3 \mathrm{~cm}$ superiorly, the anterior superior iliac spine laterally, and the pubic symphysis medially (across its middle line) and be inserted into the suprapubic space rather than directly over the bladder. Whether to fix the mesh remains controversial, though it is recommended for beginners to fix the mesh (Figure 18). The mesh is fixed using spiral tacks, starting with a tack placed through the mesh into the Cooper ligament. This placement is made with care to avoid damaging the Corona Mortis vessels and the external iliac artery. When the medial edge of the mesh is secured with one tack, it is easier to continue to fix the mesh anteriorly to the pubic tubercle, superior-laterally to the conjoint tendon and the dorsal aspect of the rectus abdominis. Care should be taken to avoid involvement of the inferior epigastric vessels lateral to the rectus abdominis. The bleeders caused by tack placement can be directly ligated or clipped. For direct inguinal hernias, fixations of the mesh to the pubic tubercle and the Cooper ligament are very important. Specific attention should be paid to the tack placement on the lateral-superior corner of the mesh. First, the tack placement cannot be lower than the level of the iliopubic tract to prevent damage and entrapment of the lateral femoral cutaneous nerve; second, the tack should not be forced through the muscle to prevent entrapment of the iliogroinal or iliohypogastric nerve; finally, it is important to ensure that the lateral corner of the mesh lies flat and that the peritoneal folds and the hernia sac are positioned on the top of the mesh to prevent herniation of the retroperitoneal fat tab, the bowel loops and the hernia sac beneath the mesh. Generally speaking, fixation of the mesh into the Cooper ligament and the conjoint tendons and fixation of the lateral corner are sufficient to prevent hernia recurrence. To reduce postoperative pain caused by spiral tacks, the alternatives are to use biological glue to fixate the mesh or to use no fixation at all. The technical rationale for such an approach is to use a large mesh $\left(15 \times 12-\mathrm{cm}^{2}\right.$ mesh) for abdominal hernia with a hernia ring diameter $<3 \mathrm{~cm}$ or to use 3D mesh designed to conform to the inguinal anatomy. The use of self-fixating mesh is also an option, as it can be fixed to the inguinal area via microgrips made of resorbable polylactic acid (Figure 19).

Peritoneal closure. The free peritoneal edges are grasped and brought up. If greater tension exists, reducing the intraabdominal pressure could help to align the upper edge with the lower edge. A suture or interrupted stapling can be used to close the peritoneum in the peritoneal cavity. Endoscopic suture can be used for fast closure. Stapling principles are as follows: (I) protect the inferior epigastric vessels; (II) stapling is prohibited below the level of the iliopubic tract; and (III) the polypropylene mesh must be completely covered by the peritoneum; the peritoneal incision is closed completely to prevent postoperative adhesions, bowel obstruction or erosion.

The abdominal cavity is examined to identify any bleeders and side injury; the pneumoperitoneum can be released, and the $10-\mathrm{mm}$ trocar site is closed.

\section{Laparoscopic TEP inguinal hernia repair (Figure 20)}

Routine preparation, draping and abdominal cavity exploration. A 1.5 -cm curved infraumbilical incision is made, and a small retractor is used to assist with the exposure. Only one incision in the rectus sheath is made for conventional TEP repair, and no abdominal exploration is required. Combining conventional TEP with abdominal exploration can integrate many advantages of the TAPP approach. The surgical details are as follows: the anterior rectus sheath is dissected along the middle line (1-cm width to each side). A $0.6-\mathrm{cm}$ transverse incision is made in each side of the middle line (Figure 21). A Veress needle is inserted in the contralateral rectus sheath to establish pneumoperitoneum with pressure maintained at $12-14 \mathrm{mmHg}$. Then, a $10-\mathrm{mm}$ trocar and a $30^{\circ}$ laparoscope are placed through this incision to explore the bilateral inguinal region, to reduce the hernia contents, and to confirm the diagnosis and surgical plan (Figure 21, showing a right indirect inguinal hernia). The intraperitoneal gas is then allowed to escape, the trocar is 


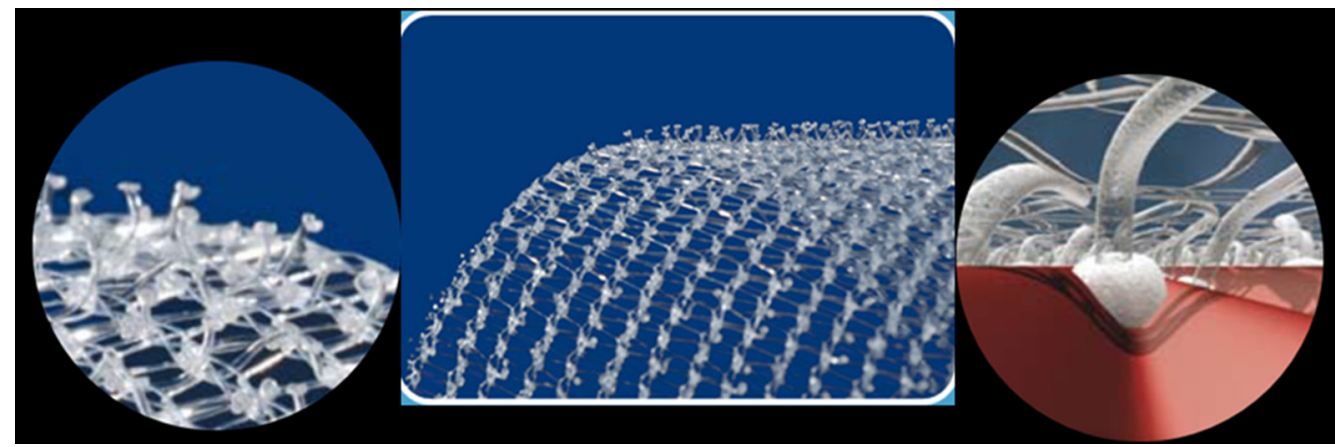

Figure 19 Schematic of self-fixating mesh. Resorbable polylactic acid microgrips are evenly distributed on one side of the mesh. The absorption time is 15 months. The tip of a microgrip is round and dull; the microgrips are embedded in rather than penetrate the tissue, without damaging the nerves, and are automatically fixated).

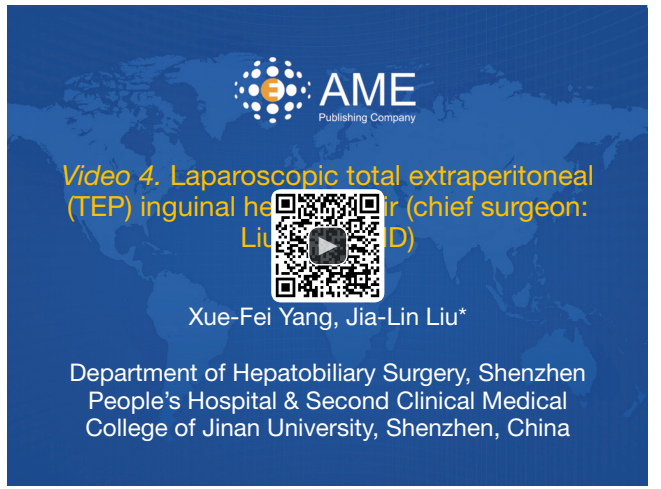

Figure 20 Laparoscopic total extraperitoneal (TEP) inguinal hernia repair (chief surgeon: Liu Jialin, MD) (8).

Available online: http://www.asvide.com/articles/1177

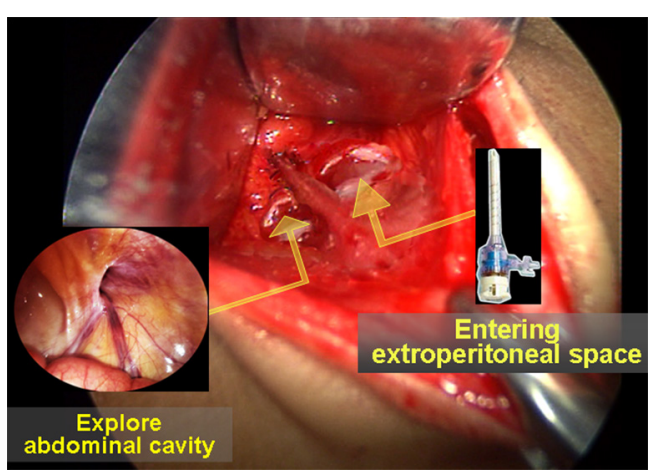

Figure 21 Combining abdominal exploration with the total extraperitoneal (TEP) approach.

removed, and the incision in the anterior rectus sheath for abdominal exploration is closed.

Dissection of the retropubic preperitoneal space. The following procedures are the same as those employed in the conventional TEP approach. A hemostat is used to bring up the lower edge of the ipsilateral anterior rectus sheath, and the rectus abdominis fibers are retracted laterally to expose the posterior sheath. A $10-\mathrm{mm}$ trocar is inserted for $3-5 \mathrm{~cm}$ between the muscle and the posterior rectus sheath. A pneumoperitoneum tube is connected to this trocar, with the pressure maintained at $12-14 \mathrm{mmHg}$. A $10-\mathrm{mm} 30^{\circ}$ laparoscope is placed through this trocar, and the dissection is continued to expose the retropubic extraperitoneal space. A variety of methods are available to dissect this space, including balloon dissection and blunt dissection with the fingers or the laparoscope. In this chapter, we focus on blunt dissection with the laparoscope. The surgeon puts one hand on the external abdominal wall for guidance of the laparoscope and uses the other hand to hold the laparoscope, under direct laparoscopic visualization, to extend toward the pubic symphysis between the superficial and deep transverse fascia and then to penetrate through the deep transverse fascia into the space of Retzius. At this stage, the featured web-like loose connective tissues are visualized. In this maneuver, care should be taken to avoid pushing the laparoscope either too deeply into the abdominal cavity or too superficially into the subcutaneous layer (Figure 22). When the laparoscopic tip reaches the pubic symphysis, the laparoscope is then moved slightly side-to-side to expand the dissection of the space of Retzius; the pubic symphysis and the bright-white Cooper ligament can be initially exposed at this stage. The maneuver should be performed gently to avoid piercing the peritoneum. The range of the dissection should be carefully controlled to avoid extending to the anterior region of the pubic symphysis or the region 


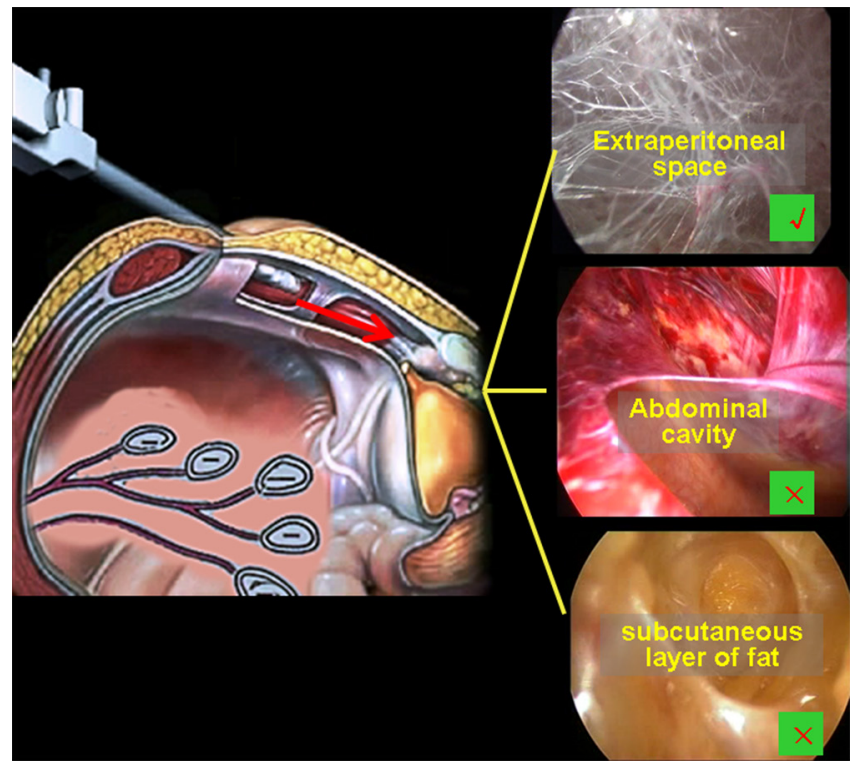

Figure 22 Gaining correct access to the space of Retzius.

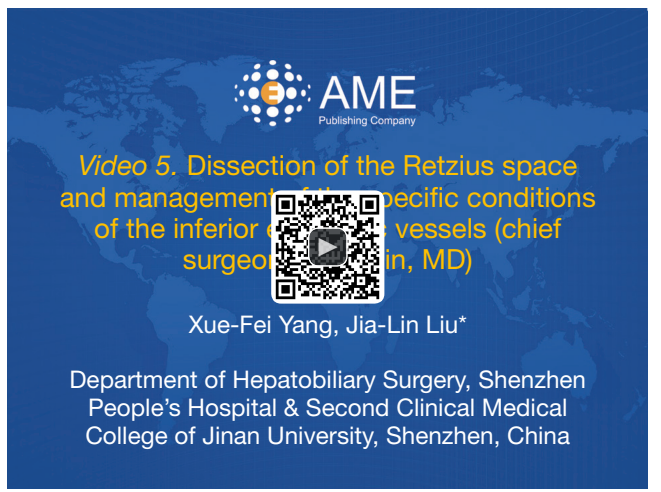

Figure 23 Dissection of the Retzius space and management of the specific conditions of the inferior epigastric vessels (chief surgeon: Liu Jialin, MD) (9).

Available online: http://www.asvide.com/articles/1179

$5 \mathrm{~cm}$ lateral to it to prevent damaging the posterior vein of the pubic symphysis, the Corona Mortis vessels, and the external iliac vessels or the inferior epigastric vessels (Figure 23). To reduce air leakage around the $10-\mathrm{mm}$ trocar, a pre-suture can be placed around the umbilical incision and tied tightly after completion of the dissection of the Retzius space. A dissecting balloon trocar can be used to achieve a better result; however, this procedure is more costly;

After the space of Retzius is dissected using the laparoscope, two 5-mm trocars are placed through the predetermined sites to provide access ports for a dissecting clamp and a pair of scissors. If necessary and when appropriate, the transverse fascia and the semicircular line near the laparoscopic trocar port can be separated to improve surgical visualization. With combined blunt and sharp dissection, the deep transverse fascia is incised along the pubic symphysis pubis and bilateral pubic ramus to completely expose the pubic symphysis, the pubic tubercle, the ipsilateral Cooper ligament and the space of Retzius. At this stage, the surgeon should be aware that a direct hernia sac could pass over the pubic ramus and the Cooper ligament into the Hesselbach triangle. The dissection is continued laterally along the Cooper ligament to a site $5 \mathrm{~cm}$ away from the pubic symphysis, where the Corona Mortis vessels and the femoral ring anterior-superior to the Corona Mortis vessels can be visualized. Attention should be paid to identify whether a femoral hernia sac protrudes through the femoral ring. As the Cooper ligament is further exposed, the external iliac artery and vein come into view and run straight-inferiorly to cover the Cooper ligament. It is easy to identify the inferior epigastric artery, which is located anterior-superior to the external iliac vessels. If any indirect inguinal hernia sac is present, the vas deferens and the testicular blood vessels, which are covered by the internal spermatic cord fascia, may be visualized between the inferior epigastric vessels and the external iliac vessels;

Dissection of the space of Bogros and the iliac fossa. The deep abdominal transverse fascia is thick and dense in approximately $50 \%$ of patients. It extends laterally to fuse with the anterior abdominal wall (Figure 24). However, the fusion becomes loose at the internal ring. Thus, it is easy to dissect the space of Bogros starting from the internal ring. A dissecting clamp is placed just behind the inferior epigastric vessels to dissect the space between the vessels and the indirect inguinal hernia sac or the peritoneum and then eventually to access the space of Bogros (Figure 25). In some patients, the fusion portion between the abdominal transverse fascia and the peritoneum is very dense; peritoneal penetration is likely to occur during the dissection, causing $\mathrm{CO}_{2}$ into the abdominal cavity. Therefore, care must be taken to avoid involving the peritoneum when incising tough and thickened transverse fascia (Figure 26). A non-dissected inguinal hernia sac will likely pull the peritoneum to cover the internal ring. This condition limits the expansion of the operative field. Therefore, it is difficult to completely dissect the space of Bogros and the iliac fossa before dissecting the hernia sac. At this stage, the space of Bogros only needs to be slightly developed to facilitate dissection of the hernia sac 


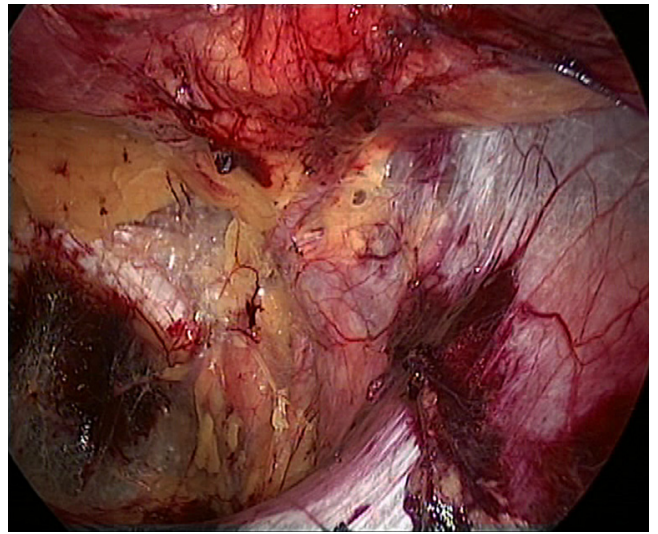

Figure 24 The deep transverse abdominal fascia is tightly attached to the anterior abdominal wall at the site lateral to the inferior epigastric blood vessel.

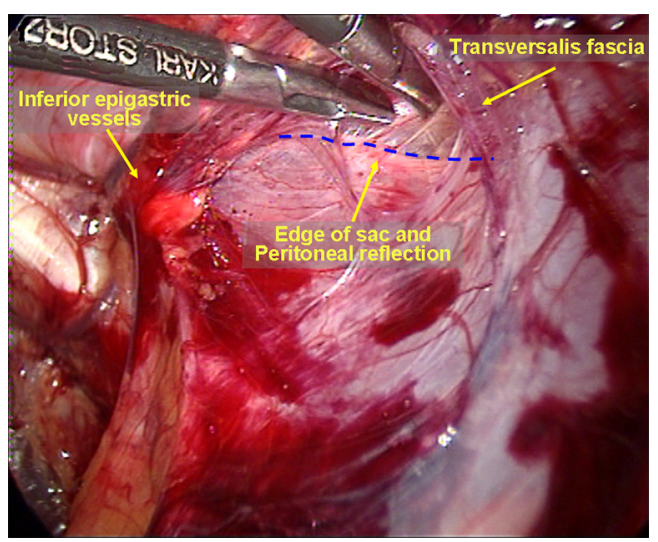

Figure 25 Accessing the space of Bogros.

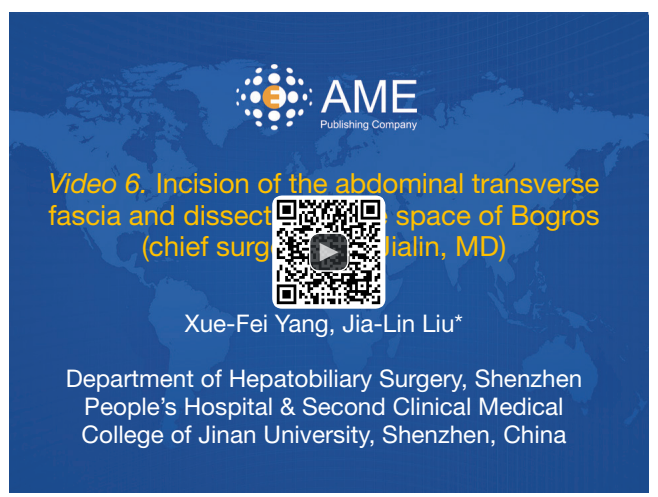

Figure 26 Incision of the abdominal transverse fascia and dissection of the space of Bogros (chief surgeon: Liu Jialin, MD) (10). Available online: http://www.asvide.com/articles/1180
(Figure 27).

Dissection of the hernia sac. Dissection of the direct hernia sac is very straightforward, as previously described in the section summarizing the TAPP approach (Figure 28). To avoid postoperative seroma after direct inguinal hernia repair, the loose abdominal transverse fascia will be pulled out of the abdominal wall defect; then, it can either be suture ligated or tacked to the Cooper ligament to make the abdominal wall defect smaller or even to make it disappear; these operations are believed to reduce the likelihood for postoperative hematoma and seroma formation. Indirect hernia sacs can be more difficult to dissect, depending on the hernia sac diameter, thickness, depth, and adhesive degree around the internal ring. The next steps are to incise the internal spermatic fascia and to expose the white sac, after which the hernia is separated free from the vas deferens and the testicular vessels by blunt dissection. It is not necessary to intentionally dissect the base of hernia sacs. The hernia sac is ligated and transected after dissection around the sac at the level of the internal ring; the distal end is left in situ. Hemostasis is essential for the sac stumps.

Parietalization of cord structures and complete dissection of the space of Bogros and the iliac fossa. A dissecting clamp is used to grasp and pull the stump of an indirect hernia sac in a cephalad direction while the other clamp is used to separate the testicular vessels and the vas deferens from the sac. The fat tabs attached to the spermatic cord do not need to be separated from the spermatic cord. At this point, the testicular vessels and the vas deferens become part of the abdominal wall. The vas deferens is often surrounded by the deep transverse fascia (preperitoneal loop), which needs to be transected to make the vas deferens long enough and to ensure that the distance between the hernia sac or the peritoneal fold and the root of the inferior epigastric vessels is greater than $5 \mathrm{~cm}$. This step will provide ample space for placement of the mesh. The distance between the hernia sac or the peritoneal fold and the root of the inferior epigastric vessels, i.e., the distance of the separated space from the internal ring, is greater than $5 \mathrm{~cm}$; this step will provide ample space for placement of the mesh. In patients with direct inguinal hernia, it is important to identify the internal ring and the site of the peritoneal fold by pulling the hernia sac in a cephalad direction. The dissection now continues toward the lateral space along the peritoneal fold to completely separate the space of Bogros and the iliac fossa until reaching the anterior superior iliac 


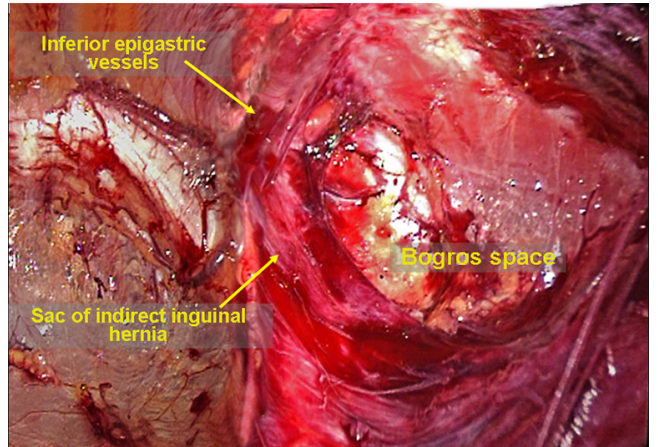

Figure 27 Initial dissection of the space of Bogros.

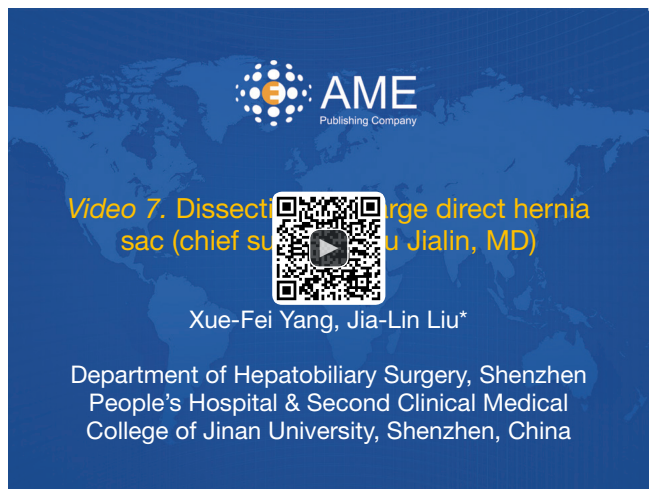

Figure 28 Dissection of a large direct hernia sac (chief surgeon: Liu Jialin, MD) (11).

Available online: http://www.asvide.com/articles/1181

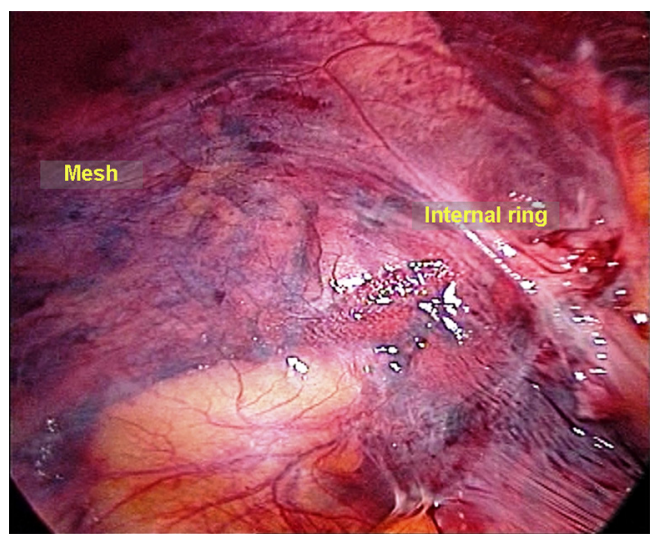

Figure 29 Abdominal re-exploration at the end of the procedure, if necessary.

spine; the deep abdominal transverse fascia surrounding the peritoneal fold should be transected to make the dissection easier. Occasionally, large fat tabs attached to the posterior wall of the space of Bogros impede the dissection. It is important to patiently sweep these fat tabs toward the cephalad side. Care should be taken to protect the iliac fascia to avoid damaging the genitofemoral nerve and the lateral femoral cutaneous nerve;

Tailoring, placement and fixation of the mesh. The size and shape of the mesh were described previously in the section summarizing the TAPP procedure. The mesh is then placed through the $10-\mathrm{mm}$ trocar into the extraperitoneal space. The mesh roll is unrolled and made to lie flat. The mesh should cover the pubic symphysis for $2-3 \mathrm{~cm}$ medially, the anterior superior iliac spine laterally, and the pubic ramus medial-inferiorly. It is important to ensure that the mesh covers the myopectineal orifice adequately. The lateral portion of the mesh is unfolded flat; one should ensure that the lower edge of the mesh attaches to the peritoneal fold. The procedure for the fixation of the mesh is the same as described in the section summarizing the TAPP procedure. If a self-fixating mesh is used, it should be wrapped with a film paper because it is difficult to adjust its position once it attaches to the tissue. The film paper can be removed after the mesh is placed and unfolded successfully (Figure 20).

Drainage tube placement. Whether to place a drainage tube is based on the conditions of the operative field. In the settings of a giant hernia sac, recurrent hernia, moderate bleeding and the early stage of the "learning curve" for the TEP procedure, it would be better to place an extraperitoneal drainage tube posterior to the pubic symphysis that can be removed in 24 to 48 hours, or if necessary, in 72 hours.

End of the procedure. A dissecting clamp is used to pin the mesh (on the lateral-inferior corner) against the psoas muscle and to eliminate the potential space for herniation between the lower edge of the mesh and the psoas muscle. When the pneumopreperitoneum is slowly released, the peritoneum spontaneously sits on the mesh due to the intra-abdominal pressure. If operations such as reduction of the greater omentum via the abdominal cavity and peritoneal rupture repair are performed at the beginning of surgery, it is reasonable to explore whether active bleeding of the omentum, peritoneal rupture, and mesh migration are present through the original umbilical exploration incision. The incisions in the anterior rectus sheath at each side of the midline are closed at the end of the procedure (Figure 29). 

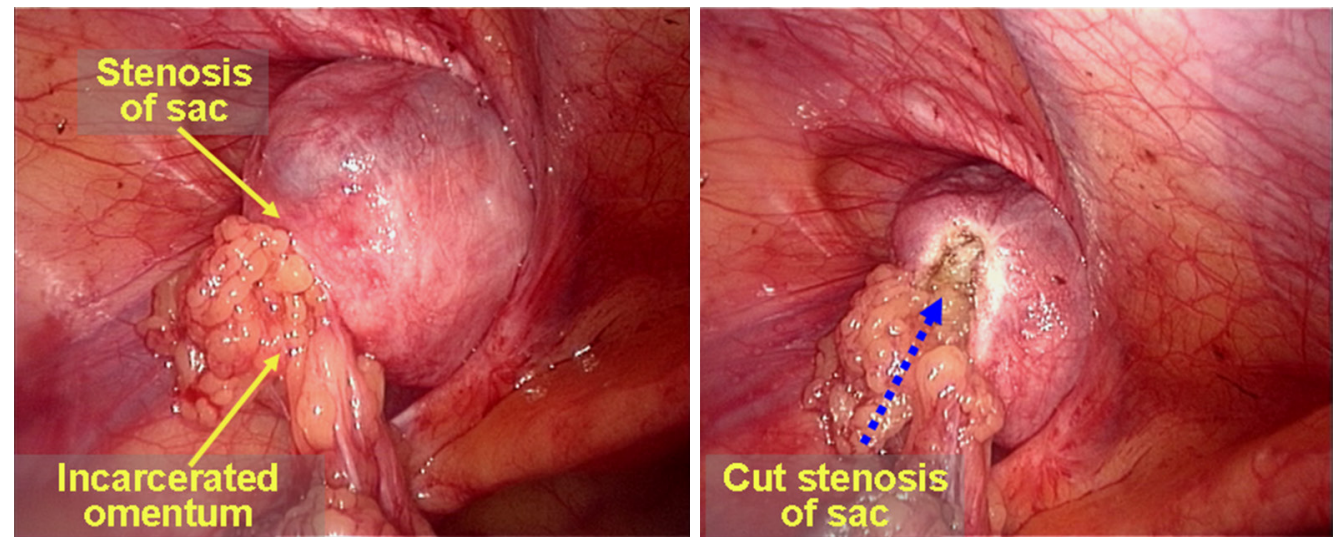

Figure 30 Management of the incarcerated omentum.

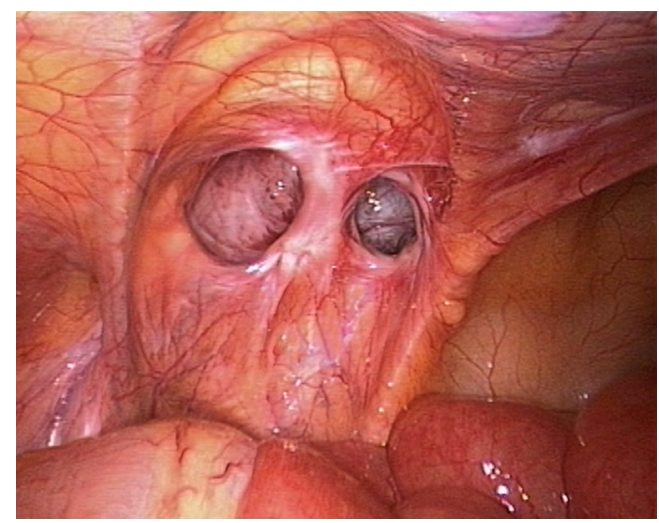

Figure 31 A prolonged direct hernia sac extending into the scrotum.

\section{Special conditions and management}

Difficult reduction of the greater omentum. The greater omentum is the most common content in hernia sacs. In general, the surgeon may attempt to use a hand to press the groin region to reduce the omentum back into the abdominal cavity if there are no adhesions or if only mild adhesions are present between the hernia sac and the omentum. A dissecting clamp is needed to help with the reduction of the omentum if there is severe adhesion between the hernia sac and the omentum or severe incarcerated hernia. In the IPOM and TAPP procedures, the reduction can be completed with routine trocars and a dissecting clamp, while an additional $5-\mathrm{mm}$ trocar via a para-umbilical incision at the lateral edge of the rectus abdominis in the affected side is needed in the TEP procedure. During the reduction, the omentum can be reduced by cutting it into several pieces with a hand pressing on the external abdominal wall simultaneously. If the hernia ring stricture is evident, the upper edge of the hernia sac or the hernia ring can be incised for 1-2 cm using a diathermy hook or scissors, with care taken to protect the interior epigastric vessels. This procedure is effective in most cases (Figure 30). If the intra-abdominal reduction fails, a small auxiliary incision can be made in the groin region to perform an open reduction or to remove the incarcerated omentum, after which the hernia sac can be dissected and ligated. The TEP or TAPP procedure can continue after the incision is closed in layers, or the surgery can be converted to a direct open hernia repair.

Complex direct inguinal hernia. In laparoscopic repair, the dissection of a direct hernia sac is generally easier than that of an indirect inguinal hernia sac. The same is true for the dissection of the internal ring and the peritoneal folds. However, complex direct hernias, such as a multiple unilateral direct hernia or a prolonged direct hernia sac that extends into the scrotum (Figure 31), cannot be separated using conventional approaches because of the severe adhesions around the sac. However, these structures can be ligated and transected at the hernia ring, as in the management of indirect hernias, which makes the procedure significantly easier.

Cryptorchidism and complicated indirect inguinal hernia. Adult cryptorchidism complicated with indirect inguinal hernia accounts for approximately $25.6 \%$ of patients with cryptorchidism. Laparoscopic inguinal hernia repair and laparoscopic orchiectomy for an undescended testicle can be combined to complete the treatments in the same procedure. The type of laparoscopic inguinal hernia repair 

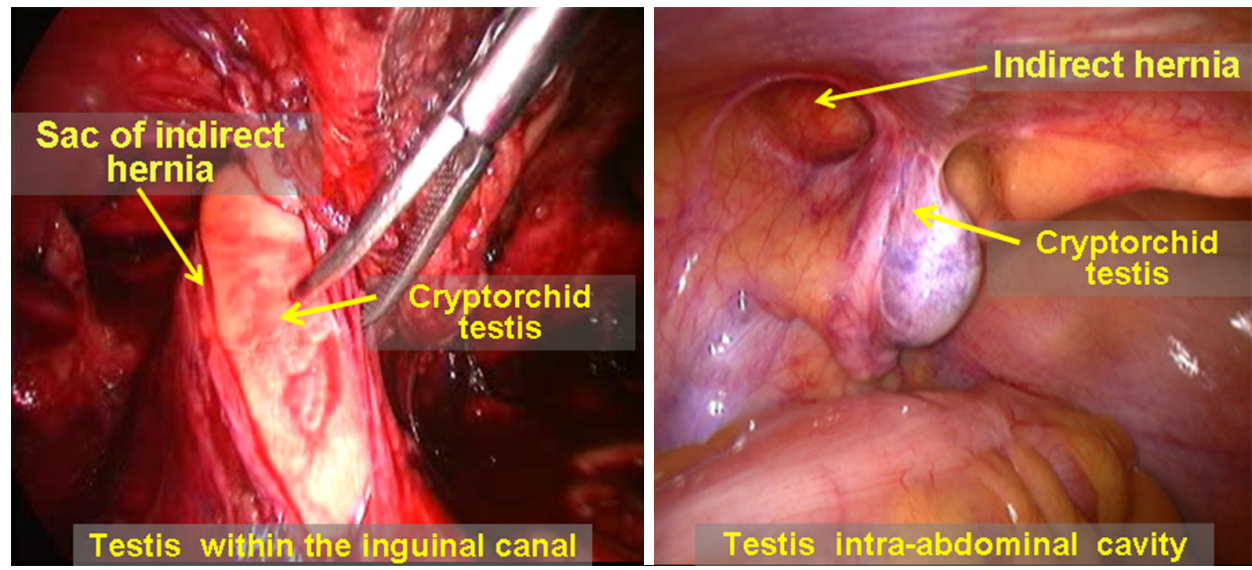

Figure 32 Cryptorchid testis in inguinal canal and intra-abdominal cryptorchid testis complicated with indirect inguinal hernia.

that should be used depends on the type of cryptorchidism, whether correction or removal of the undescended testicle is chosen as a surgical course and the presence of intact peritoneum in the inguinal region. For patients with intrainguinal canal cryptorchid testis retention, using the TEP approach requires a more experienced hand than using the TAPP or IPOM approaches. For patients with intraabdominal cryptorchid testis retention, the TEP approach can be used to complete the hernia repair after laparoscopic orchiectomy for the undescended testicle if removal is planned. The IPOM approach is an optimal selection in patients who will undergo orchidopexy. The trocar should be placed in the lateral edge of the rectus abdominis at the level of the umbilicus (Figure 32).

Bleeding in the operative field. During adult laparoscopic inguinal hernia repair, there are several common sources of bleeding. (I) Peripheral vessels of the injured pubic ramus: the venous blood loss is more severe than the arterial blood loss; the surgeon should be careful to perform diathermy coagulation or bipolar coagulation, if necessary; (II) injured retropubic venous plexus: the retropubic venous plexus drains toward the perineum into the dorsal penile venous plexus in which the vessels are thicker, larger and clustered with considerable blood flow. At the dissection of the space between the pubic symphysis and the bladder, care must be taken not to separate too deeply; bleeding should be managed with gauze compression immediately. Suction is used to remove the blood, and bipolar diathermy may be used to control bleeding. Suturing may be used to stop bleeding if necessary; (III) injured inferior epigastric vessels and their branches: such vessels should be either clipped or ligated. In particular, if the inferior epigastric vessels are injured during dissection of the rectus abdominis around the trocar, the significantly pulsatile bleeding can be seen from a branch of the inferior epigastric artery. Bipolar diathermy or titanium clips can be used for bleeding control, if necessary; (IV) injured small vessels in the abdominal transverse fascia during dissection of the space of Bogros: it is easy to control bleeding in this case; $(\mathrm{V})$ torn small vessels around the spermatic cord during dissection of an indirect hernia sac: bleeding from these vessels is self-limited; (VI) torn Corona Mortis vessels: bleeding from these vessels is difficult to control due to the vulnerable vessel wall and its running path (through the femoral ring). A small gauze can be placed with pressure for 3-5 minutes; once the bleeding has slowed, a bipolar diathermy can be used for hemostasis as soon as suction is applied to expose the bleeders.

Tear of the peritoneum. Tearing of the peritoneum during a TEP procedure is quite common. A tiny tear does not require treatment. If $\mathrm{CO}_{2}$ enters the abdominal cavity from the extraperitoneal space and affects the operative field, a Veress needle can be used to release $\mathrm{CO}_{2}$ through the umbilical incision to maintain adequate extraperitoneal space for the surgery. Larger tears $(>1 \mathrm{~cm})$ will not only cause difficulty in the dissection but will also induce postoperative adhesions. Therefore, larger tears can be grasped with a dissecting clamp and then ligated, clipped, or sutured (including continuous suture) for closure.

Accidental dissection of the inferior epigastric artery. The inferior epigastric vessels can be accidentally freed from the abdominal wall during dissection of the space posterior to the pubic bone and the groin. These vessels then hang in the middle of the operative field and affect the surgery. To this end, care should be taken to identify the inferior gastric 
vessels and to avoid separating them accidently. However, if separation occurs, the remedy is to pull and fix the vessels to the abdominal wall with a suture (through the abdominal wall) or a dissecting clamp to ensure a smooth procedure.

Unexpected sac contents. Even though the preoperative physical examination should indicate complete reduction of the hernia contents, ectopic adhesive omentum, the appendix, the fallopian tubes, and the ovaries may be still present in the inguinal canal in some patients (Figure 33). Complete reduction of the contents can only be determined during a laparoscopic procedure.

Hernia recurrence after tension-free inguinal hernia repair (Figure 34). It is not necessary to dissect or remove the polypropylene mesh due to dense adhesions between the patient's tissue and the mesh. Previously implanted mesh can be considered as part of body tissue. If removal of previously placed polypropylene mesh is indicated, a diathermy hook and scissors can be used. The TEP, TAPP,

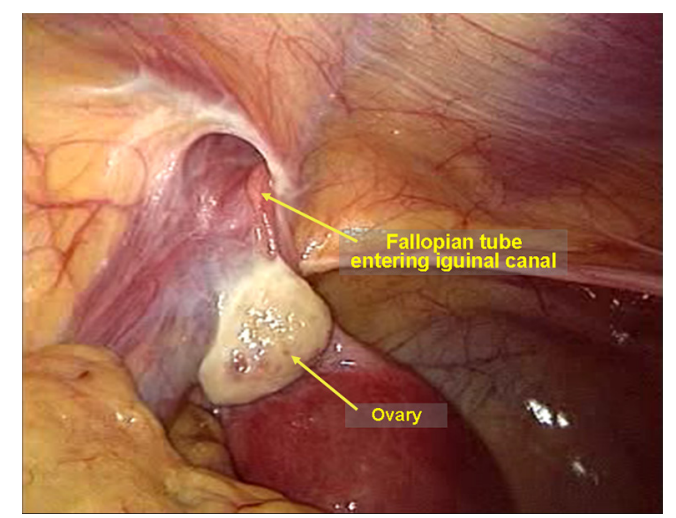

Figure 33 The ampulla of the left fallopian tube is present in the inguinal canal. and IPOM approaches can be selected for additional laparoscopic surgical repair according to the degree of adhesions in the abdominal extraperitoneal space caused by the previous surgery. The TAPP approach is recommended as a first line procedure.

Drainage tube placement. Drainage tube placement is not required in the IPOM and TAPP approaches. In contrast, it is beneficial to place a drainage tube after the TEP approach because the extraperitoneal space is relatively small, and the accumulation of blood or fluid can lead to infection and mesh migration, particularly in patients with significant bleeding during surgery. In general, postoperative bleeding in the operative field is associated with severe adhesions around the sac or a larger sac. In patients with complete giant inguino-scrotal hernia, it is necessary not only to place an extraperitoneal drainage tube but also to include an isolated tube for the residual sac drainage. The indwelling time of the drainage tube depends on the daily drainage volume. The tube can be removed when the drainage is clear and its volume is less than $5 \mathrm{~mL} /$ day, which may take 24 to 48 hours and occasionally as long as 72 hours.

Trusses are recommended for patients with giant inguino-scrotal hernia. In patients with huge inguinal hernias (size of the hernia sac $>15 \mathrm{~cm}$ ), the defect of the internal ring is relatively large (diameter $>4 \mathrm{~cm}$ ). The treatment for abdominal incisional hernia should be considered as a reference. Hernia may be recurrent in these patients when they experience an abrupt increase of intra-abdominal pressure during postoperative anesthesia recovery. The trusses should be dressed at the end of surgery before awakening. The belt may be removed 14-21 days after surgery when the mesh has firmly fused with the surrounding tissue. This management can help to
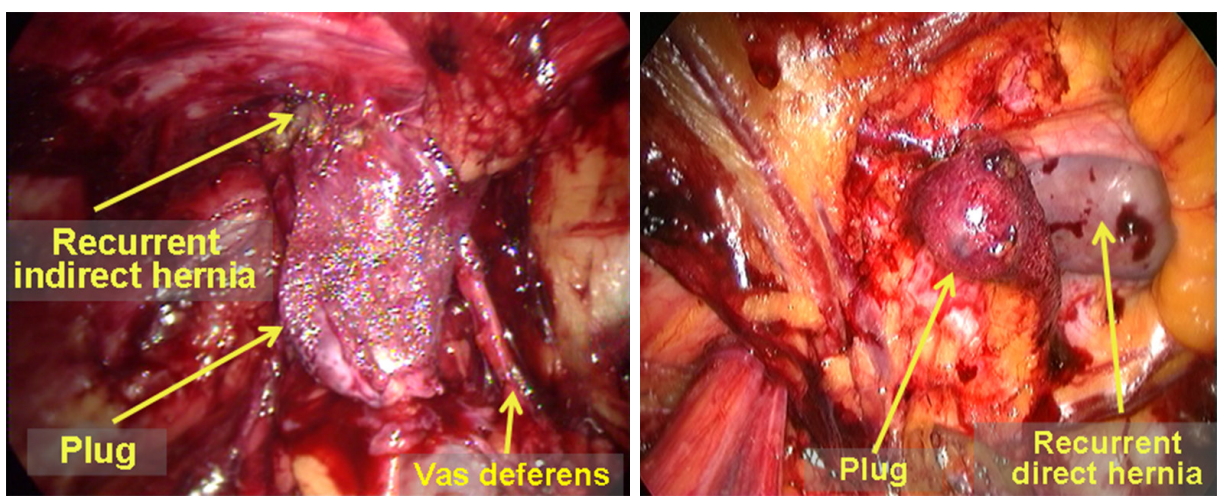

Figure 34 Hernia recurrence after left indirect and direct inguinal hernia repair. 
maintain the effectiveness of the hernia repair.

Hematoma or seroma. Postoperative hematoma or seroma often occurs in the groin area or the distal residual sac in the scrotum. Large cases may be misdiagnosed as recurrent hernia, and ultrasound examination can be helpful for the differential diagnosis. Postoperative hematoma is commonly associated with bleeding in the operative field or incomplete drainage, while seroma is associated with stimulation of the surrounding tissue by the artificial mesh to produce more exudate. Generally speaking, the volumes of hematomas and seromas are usually not excessive; therefore, no special management is needed. The majority of seromas can be absorbed spontaneously in a month and disappear within 3 months of surgery; the fluid can be aspirated under ultrasound guidance a week after surgery if the hematoma or seroma is relatively large. Hematomas can disappear after 1 or 2 aspirations, while seromas may

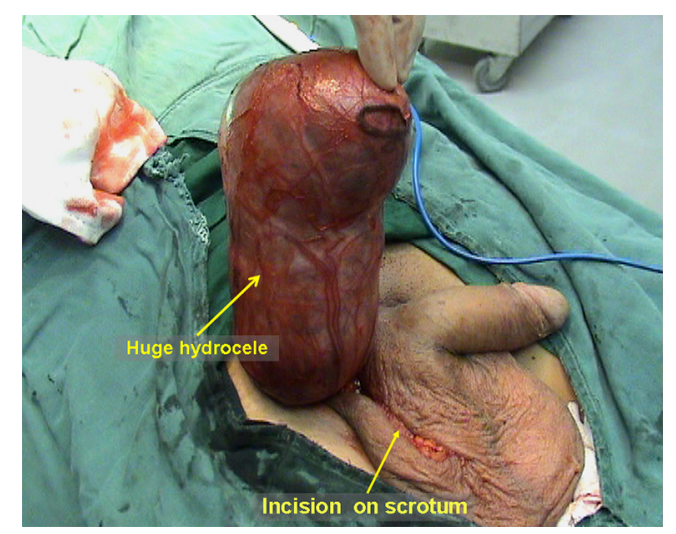

Figure 35 Postoperative hydroceles after total extraperitoneal (TEP) for right indirect inguinal hernia.
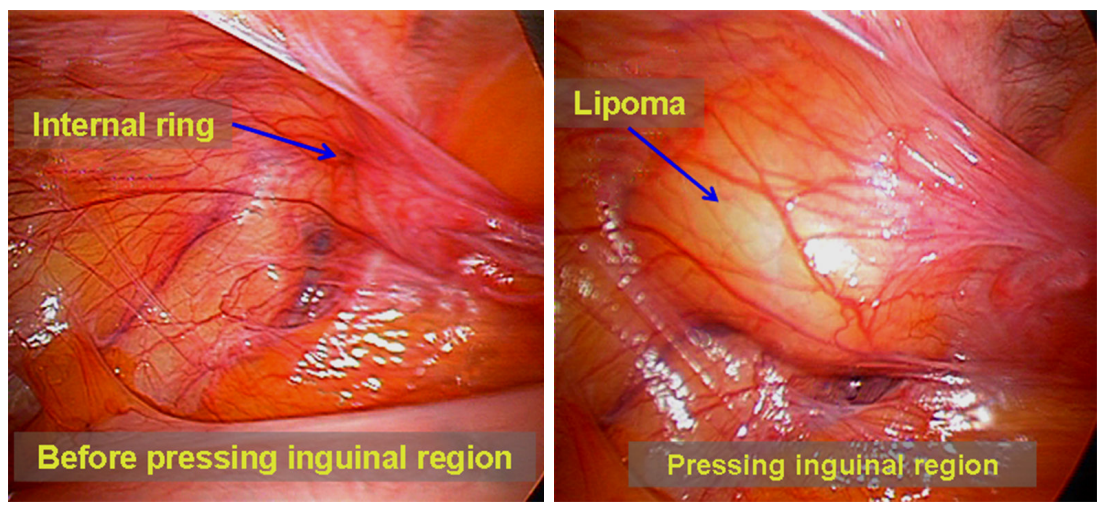

Figure 36 Spermatic cord lipoma is difficult to diagnose before surgery. require more aspirations. In a few patients with indirect inguinal hernia, postoperative delay hydrocele may present in the distal residual hernia sac. Eversion of the tunica vaginalis as a remedy for hydrocele may be needed if 3 -month conservative treatments, such as physical therapy and aspiration, are ineffective (Figure 35).

Negative exploration. Occasionally, laparoscopic exploration cannot find the internal ring defect, even though an inguinal hernia was diagnosed before surgery in clinical practice. This failure should not immediately terminate the surgery; spermatic cord lipomas or femoral hernias should be taken into account, as they are difficult to be confirmed before surgery. One may find that a spermatic cord lipoma protrudes into the abdominal cavity via the inguinal canal when using a hand to press the groin region on the external abdominal wall (Figure 36). Spermatic cord lipomas can often result in defects of the inguinal canal and are also a cause of indirect inguinal hernia. Spermatic cord lipoma excision and inguinal hernia repair must be performed spontaneously. The abdominal defect for femoral hernia is usually small. Thus, careful examination should be performed to exclude femoral hernia.

Management of a sliding hernia. The posterior wall of a sliding indirect inguinal hernia sac is usually the ileocecal portion (Figure 37), though it may be the bladder in a few cases. The IPOM or TAPP approach can be used to expose the inguinal region and to repair the myopectineal orifice, following reduction of the hernia content by pressing the external abdominal wall. Fixation of the mesh is very important in this case because sliding hernia is commonly present as a huge scrotal hernia with a larger internal ring. Following surgery, wearing trusses are recommended immediately and for 2 to 3 weeks after to ensure complete fusion of the mesh and the surrounding tissue. 

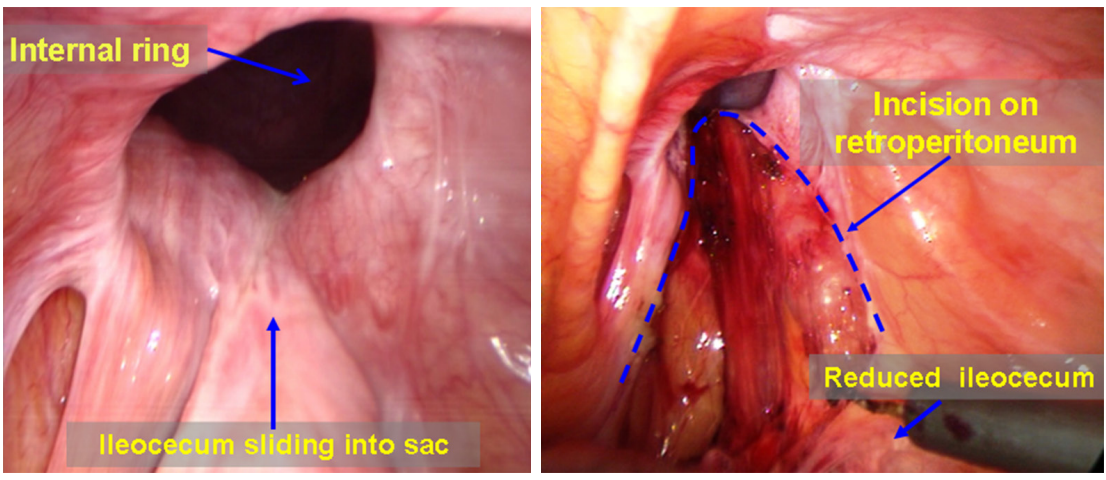

Figure 37 Management of a sliding hernia.

Conversion to open surgery. When insurmountable difficulties occur during laparoscopic inguinal hernia repair, conversion to open surgery should take place immediately. Conversion to open surgery is very common when performing the TEP approach. For example, conversion from a TEP to a TAPP procedure would be required when extensive tears to the peritoneum during a TEP procedure cannot be repaired. After completion of the TAPP procedure, the peritoneum rupture can be closed through the abdominal cavity. Another example is the conversion of a TAPP to an IPOM (using the anti-adhesion mesh) procedure if the peritoneum is difficult to dissect during the TAPP procedure and the polypropylene mesh cannot completely cover the affected area. Severe abdominal adhesion is an indication for conversion to open surgery. Because the abdominal transverse fascia is often torn during a laparoscopic procedure, the torn abdominal transverse fascia should first be repaired during open surgery, after which the mesh can be placed. The Lichtenstein procedure is the more convenient procedure.

Postoperative pain of the lower abdominal wall. It is very important to determine if pain is present in the groin region and the degree of pain before surgery. It is particularly important to identify whether groin pain is secondary to inguinal hernia, femoral hernia or obturator hernia and to rule out lesions of the lumbar spinal cord, the pelvis, the hip and the urogenital system, which may cause pain in the groin region. In general, varying degrees of pain (that become worse with movement) are present in the groin regions of all patients immediately after surgery due to tissue dissection and mesh fixation. The incidence of pain is higher in the TAPP and IPOM procedures. The pain is tolerable in most of cases; however, if the pain is severe, regardless of the patient's position and movements and evokes burning, electric, and tingling sensations in the regional skin, nerve damage should be considered, and electrophysiological examination is indicated. Injuries of the lateral femoral cutaneous nerve and the genital branch of the genitofemoral nerve are more common. A few cases with injuries of the femoral nerve, the ilioinguinal nerve and the iliohypogastric nerve have also been reported. Conservative treatments can be applied to patients with minor nerve injuries and provide obvious relief in the shortterm. If severe pain persists or is accompanied by significant dysfunction of skin sensation and lower extremity motor activity, immediate surgical exploration is required to remove the tacks and sutures or to excise the involved lateral femoral cutaneous nerve, the ilioinguinal nerve and the iliohypogastric nerve. If postoperative pain in the groin area begins and gradually worsens several weeks or months after surgery, nerve stimulation in the groin area due to mesh or scar contracture should be considered; non-steroidal antiinflammatory analgesic agents, neurotrophic agents, and local nerve blocks can be used. Furthermore, ultrasound or computed tomography scans should be performed in the groin region to exclude rare conditions, such as hematoma, mesh migration, missed hernia and hernia recurrence. If the conservative treatments described above are ineffective and the pain lasts more than six months, surgical exploration can be performed to remove the mesh and tacks or for resection of the involved nerves after the exclusion of psychological factors that may cause pain.

\section{Acknowledgements}

Funding: Shenzhen government funding for scientific and technical research and development (JCYJ20140414092023238). 


\section{Footnote}

Conflicts of Interest: The authors have no conflicts of interest to declare.

Informed Consent: Written informed consent was obtained from the patient for publication of this manuscript and any accompanying images.

\section{References}

1. Cavazzola LT, Rosen MJ. Laparoscopic versus open inguinal hernia repair. Surg Clin North Am 2013;93:1269-79.

2. Pahwa HS, Kumar A, Agarwal P, et al. Current trends in laparoscopic groin hernia repair: A review. World J Clin Cases 2015;3:789-92.

3. Pisanu A, Podda M, Saba A, et al. Meta-analysis and review of prospective randomized trials comparing laparoscopic and Lichtenstein techniques in recurrent inguinal hernia repair. Hernia 2015;19:355-66.

4. Li J, Ji Z, Li Y. Comparison of laparoscopic versus open procedure in the treatment of recurrent inguinal hernia: a meta-analysis of the results. Am J Surg 2014;207:602-12.

5. Yang XF, Liu JL. Laparoscopic intraperitoneal onlay mesh (IPOM) repair. Asvide 2016;3:403. Available online: http://

Cite this article as: Yang XF, Liu JL. Laparoscopic repair of inguinal hernia in adults. Ann Transl Med 2016;4(20):402. doi: 10.21037/atm.2016.10.37 www.asvide.com/articles/1174

6. Yang XF, Liu JL. Laparoscopic transabdominal preperitoneal (TAPP) inguinal hernia repair (chief surgeon: Liu Jialin, MD). Asvide 2016;3:404. Available online: http://www.asvide.com/articles/1175

7. Yang XF, Liu JL. Management of the round ligament of the uterus during total extraperitoneal (TEP) repair (chief surgeon: Liu Jialin, MD). Asvide 2016;3:405. Available online: http://www.asvide.com/articles/1176

8. Yang XF, Liu JL. Laparoscopic total extraperitoneal (TEP) inguinal hernia repair (chief surgeon: Liu Jialin, MD).

Asvide 2016;3:406. Available online: http://www.asvide. com/articles/1177

9. Yang XF, Liu JL. Dissection of the Retzius space and management of the specific conditions of the inferior epigastric vessels (chief surgeon: Liu Jialin, MD). Asvide 2016;3:407. Available online: http://www.asvide.com/ articles/1179

10. Yang XF, Liu JL. Incision of the abdominal transverse fascia and dissection of the space of Bogros (chief surgeon: Liu Jialin, MD). Asvide 2016;3:408. Available online: http://www.asvide.com/articles/1180

11. Yang XF, Liu JL. Dissection of a large direct hernia sac (chief surgeon: Liu Jialin, MD). Asvide 2016;3:409. Available online: http://www.asvide.com/articles/1181 